\title{
Approach to Detecting Forest Fire by Image Processing Captured from IP Cameras
}

\author{
${ }^{1}$ Tran Quang Bao, ${ }^{2}$ Nguyen Thi Hoa \\ ${ }^{1}$ Department of Environment Management, Vietnam National University of Forestry, Vietnam \\ ${ }^{2}$ Department of Resources and Environment, Vietnam National University of Forestry at Dong Nai. \\ baofuv@vfu.edu.vn; hoanguyen.nrm@gmail.com
}

\begin{abstract}
In this paper, the results show an algorithm to detect the presence of smoke and flame using image sequences captured by Internet Protocol (IP) cameras is represented. The important characteristics of smoke such as color, motion and growth properties are employed to detect fire. For the efficient smoke and fire detection in the captured images by the IP camera, a detection algorithm must operate directly in the Discrete Cosine Transform (DCT) domain to reduce computational weigh, avoiding a complete decoding process required for algorithms that operate in spatial domain. In order to assess the possibility and the accuracy of proposed algorithm, the author used the video sequences which are captured by IP camera from control forest fire at different spatial location and levels of fire intensity. Evaluation results illustrated the efficiency of the proposed algorithm in effectively detecting forest fires with accuracy at $97 \%$.
\end{abstract}

Keywords: Forest fire; Smoke and Fire Detection; DCT; IP Camera; Images Processing

\section{Introduction}

Forest fire is a complex physiological process which includes numerous direct and indirect impacts on the atmosphere, biosphere and hydrosphere. That is one of the key drivers of major changes in term of air pollution in many parts of the world. Forest fires often occur on a large scale especially in complex mountainous terrain, intricate to access. Therefore, forest fire detection by traditional approaches is inappropriate.

In recently years, Vietnam annually has 650 forest fires which are damaged average 4.340 ha of forest. In which, it has about 3.200 ha plantation forest and approximately 1.140 ha of natural forest. In 2002, forest fires in U Minh Thuong and U Minh Ha destroyed 5.500 ha of Melaleuca forest, including 60\% of primary forest. In early 2010, forest fires in Hoang Lien - Lao Cai National Park destroyed more than 700 ha of forest. The deprivations caused by forest fires in term of economic, social and environmental are quite enormous and difficult to calculate.

Early detection of forest fires can help to alarm and prevent disasters, resulting in major human and property damage. The combustion of objects usually begins with the emission of smoke, even before igniting. Therefore, smoke is an essential element for early fires detection. The smoke characteristics depend on the chemical properties, temperature, oxygen content, etc. The color of the smoke ranges from white to white-blue when fired at low temperatures and from gray to black when the temperature rises to ignition. The most general smoke detectors are based on infrared or ultraviolet cameras. In addition, other detection techniques are based on particle analysis, temperature, relative humidity, and air transpiration. These systems will operate when smoke or fire particles are close to 
fire detection devices and unable to provide information regarding the exact location, intensity, spread, etc. [1] To provide more accurate and reliable smoke detection, some video-based detection systems have been proposed. The algorithm for detecting fire through video is based on two main characteristics of fire: flame and smoke. Most fire detection algorithms in the theory use some of the characteristics of fire and smoke, such as fire / smoke color, flaking, changing of fire area boundary.

Recently, the use of IP cameras with video surveillance has grown significantly, due to the simple IPbased monitoring system at low cost. Therefore, the use of cable systems and wireless Internet infrastructure has been widely applied [3]. Furthermore, an IP camera not only captures sequences of images but also has its own processor, memory and operating system, allowing programs to process information obtained without additional computer equipment. IP cameras can be connected to form networks that make a video surveillance system more reliable. Data is the information provided by encrypted IP cameras in many format: Motion-JPEG (MJPEG), H.264, or so on... [3].

This paper focuses on image processing techniques based on the use of smoke detection algorithms from IP camera. Proposed algorithms operate directly in the DCT domain and can be implemented on IP camera surveillance systems. This algorithm has used a number of smoke features including color, motion and spread characteristics that extracted directly from the DCT coefficient during decoding.

\section{Materials and Methodology}

\subsection{Forest fire detection algorithm for IP camera}

\subsubsection{IP camera - image receiver}

The use of IP technology for forest fire detection give a countless advantage, for example IP-camera networks can detect fire origin magnitude and propagation in more accurate manner compared with a single video surveillance system. However, to efficiently use the IP technology for fire detection purposes, the smoke detection algorithm must perform directly in the Discrete Cosine Transform (DCT) domain, because decoding (from DCT domain to spatial domain) and possible encoding (from spatial domain to DCT domain) are considerably high time consuming processes. Moreover, almost all fire detection algorithms including those proposed in $[2,5,6]$ are carried out in the spatial domain, analyzing the value of each pixel or block of pixels. Therefore, any implementation of these algorithms in IP technology requires considerably high extra processing time.

Generally, an IP camera captured sequences of images, the number of frame captured depend on each different camera, normally it can reach over 20 frames per second. In addition, has its own processor, memory and operating system, allowing loaded programs to process the captured information without the need of additional computer equipment and the information provided by IP camera is encoded data in JPEG or MJPEG format.

Additionally, the quality of images captured by IP camera is stable without disturbance by signal transmission process. For normal cameras which are set up in less interfering environment and the signal is transmitted by coaxial cable to the receiver, signal still affected by material of it's cable. Therefore, the quality of images cannot be remaining for analysis process to detect objects, particularly forest fires.

\subsubsection{Image processing to detect forest fires}

Normally, IP camera uses two basic protocols to access the images that captured from the sensor via internet: http protocol (HyperText Transfer Protocol) and rtsp protocol (Real Time Streaming 
Protocol). These protocols allow access data from IP camera in two different formats: (1) http protocol allows assess and download directly JPEG images; (2) rtsp protocol uses H.264 codec, thus the received signal need a decoder to covert it ino JPEG images. Each protocol is used depending on different IP camera. Captured images by IP camera would be sort by time and then put in image processor to detect smoke or flame or event both of these signals (see fig. i).

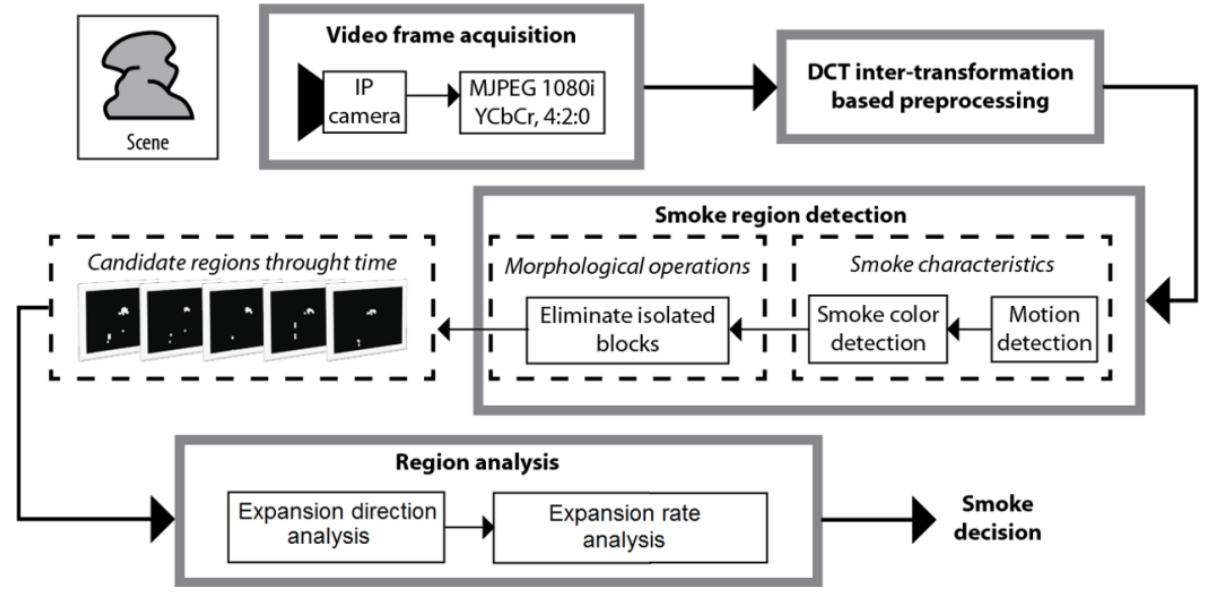

Figure 1. Block diagram of the proposed smoke detection scheme (Leonardo et al, 2012)

Captured images from IP camera usually have relatively high resolution and the minimum resolution is $1280 \times 720 \mathrm{px}$. If these images are processed directly, the processes are highly time consuming operations but high accuracy. By contrast, if these images are zoom out, the processing time requires less but low accuracy. To detect forest fire, it is not requiring high speed but need high accuracy, thus the captured images will be remaining quality for processing.

In this paper, the author not establish a new algorithm for image processing, they use the combination of many algorithms that are applying in detecting forest fire in the world [2, 4]. Including:

The image that captured by camera is divided into blocks of $8 \times 8$ pixels of each frame. After that, the DCT inter-transformation is applied to all DCT blocks of $8 \times 8$ coefficients of each frame to get DCT blocks of $4 \times 4$ coefficients. Using the DC values of each DCT block of the $4 \times 4$ coefficients of several consecutive frames, motion and color properties of smoke are analyzed to get the smoke region candidates. Each DCT block can define 3 channels including $\mathrm{Y}, \mathrm{Cb}$ and $\mathrm{Cr}$. In which, to identify the motion of DTC blocks, the author use channel $\mathrm{Y}$, and to classify the characteristics color of smoke and flame use channel $\mathrm{Cb}$ and $\mathrm{Cr}$.

- Motion detection: Consider the DC coefficients of block DCT block of $S_{b} \times S_{b}\left(S_{b}=8\right)$, this coefficient is the value to analyze the characteristics of motion of a block. $S_{b}$ times the average value of the block in spatial domain which is given by:

$$
\begin{aligned}
C(0,0)=\sqrt{\frac{2}{S_{b}}} \alpha(0) \sum_{q=0}^{S_{b}-1} & \left(\sqrt{\frac{2}{S_{b}}} \alpha(0) \sum_{q=0}^{S_{b}-1} B(p, q) \cos \left(\frac{(2 p+1) \times 0 \times \pi}{2 S_{b}}\right)\right) \cos \left(\frac{(2 p+1) \times 0 \times \pi}{2 S_{b}}\right) \\
= & S_{b} \times\left(\frac{1}{S_{b}^{2}} \sum_{q=0}^{S_{b}-1} \sum_{p=0}^{S_{b}-1} B(p, q)\right)
\end{aligned}
$$

In which, $\mathrm{Y}_{\mathrm{t}}(\mathrm{x}, \mathrm{y})$ is the DC coefficient value of a block at location $(\mathrm{x}, \mathrm{y})$ in frame $t$. Each DCT block is classified into motion or statistic block as equation below: 


$$
f_{m}\left(Y_{t-1}^{D C}(x, y), Y_{t}^{D C}(x, y)\right)=\left\{\begin{array}{c}
1 \text { if } t h_{1}<\frac{1}{s_{b}}\left|Y_{t-1}^{D C}(x, y)-Y_{t}^{D C}(x, y)\right|<t h_{2} \\
\text { otherwise }
\end{array}\right.
$$

Considering that $f_{m}$ is a binary matrix of size $M \times N(M$ is width, $N$ is the height of captured image). And th1 and th 2 are two threshold values are experimentally determined as 12 and 80 respectively which considering the general motion speed presented by smoke

-Smoke color analysis: color is another integral feature of smoke that have been used commonly in several smoke detection algorithms. Almost al algorithms used RGB and $\mathrm{YcbCr}$ (Y: Luminance; $\mathrm{Cb}$ : Chrominance-Blue; and Cr: Chrominance-Red) color space-based rules to determine smoke color.

+ These rules are given by equation:

$$
\text { Rule 1: } R \pm \alpha=G \pm \alpha=B \pm \alpha
$$

Rule 2: $80 \leq(R+G+B) / 3 \leq 220$

Where $15 \leq \alpha \leq 20$

- The pixel is considered as smoke if these adapted as follows:

Rule 1: $\left(C_{b}-128\right)^{2}+\left(C_{r}-128\right)^{2} \leq \alpha^{2}$

Rule 2: $\mathrm{Th}_{3} \leq \mathrm{Y} \leq \mathrm{Th}_{4}$

Where $C_{b}, C_{r}, Y$ are the $D C$ values of two Chrominance and Luminance of $(x, y)$ block, applying the linear transform between RGB and $\mathrm{YCbCr}$, it follows that $\alpha=10, T h 3=80$ and $T h 4=220$. Thus, if both rules are satisfied, DCT block is considered as smoke by color property, that is $f_{c}=\left(Y, C_{b}, C_{r}\right)=1$, otherwise $f_{c}=\left(Y, C_{b}, C_{r}\right)=0$.

Therefore, both feature analyses are conducted, the blocks that satisfy both smoke features are considered as smoke candidate region, as follows:

$$
B_{t}=f_{m} \wedge f_{c} \wedge f_{1} .
$$

- Region analysis

After detecting smoke candidate, it necessary to eliminate some objects possess similar properties to smoke. The connection of several connection blocks is used and each candidate region is denoted by $A_{k}, k=1,2,3, \ldots K$. where $k$ means the label number. Considering that smoke has a property of continuously expansion, the corresponding of two adjacent smoke regions presents an expansion overlapping. To analyze this smoke property, each region is updated using Equation:

$$
A_{t}^{k}=\left\{\begin{array}{c}
A_{t-1}^{k} \cup A_{t}^{k}, \text { nếu } A_{t-1}^{k} \cap A_{t}^{k} \neq \varnothing \\
A_{t}^{k}, \text { otherwise }
\end{array} \quad k=1,2, \ldots, K\right.
$$

In consequence, a new image includes motion region and is labeled can be considered as smoke region. On the other hand, if captured images are not satisfied with al the rules above, it is not considered a smoke region.

\subsubsection{System architecture (Ground monitoring station)}

An overview of the ground monitoring system is illustrated in fig. ii. The system is designed as a unified block that automatically captures images around the station. 


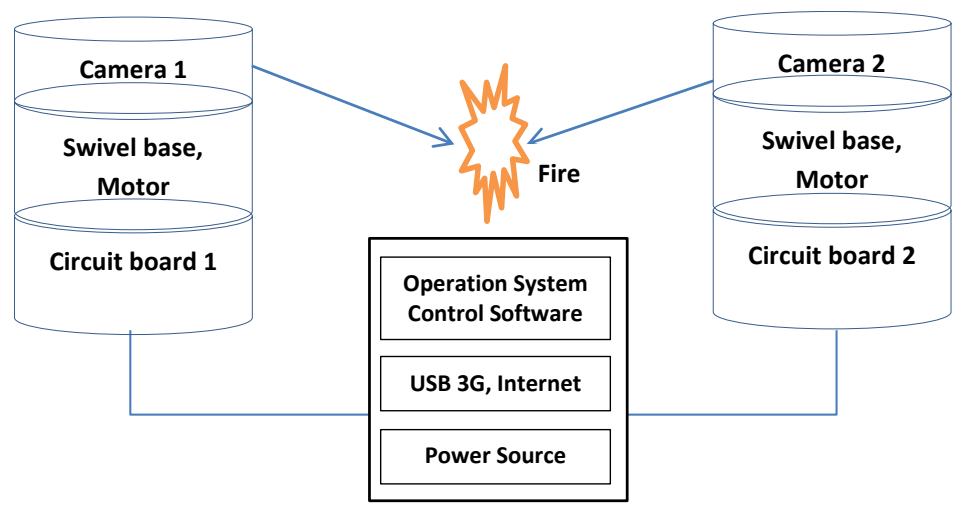

Figure 2. The block scheme of fire watch system

This system has 2 independent cameras which are rotated in a circle. All these motions are programmed to cover the area surveillance automatically by mean of repetitive sweeps. In a rotation, each camera provides 36 images evenly.

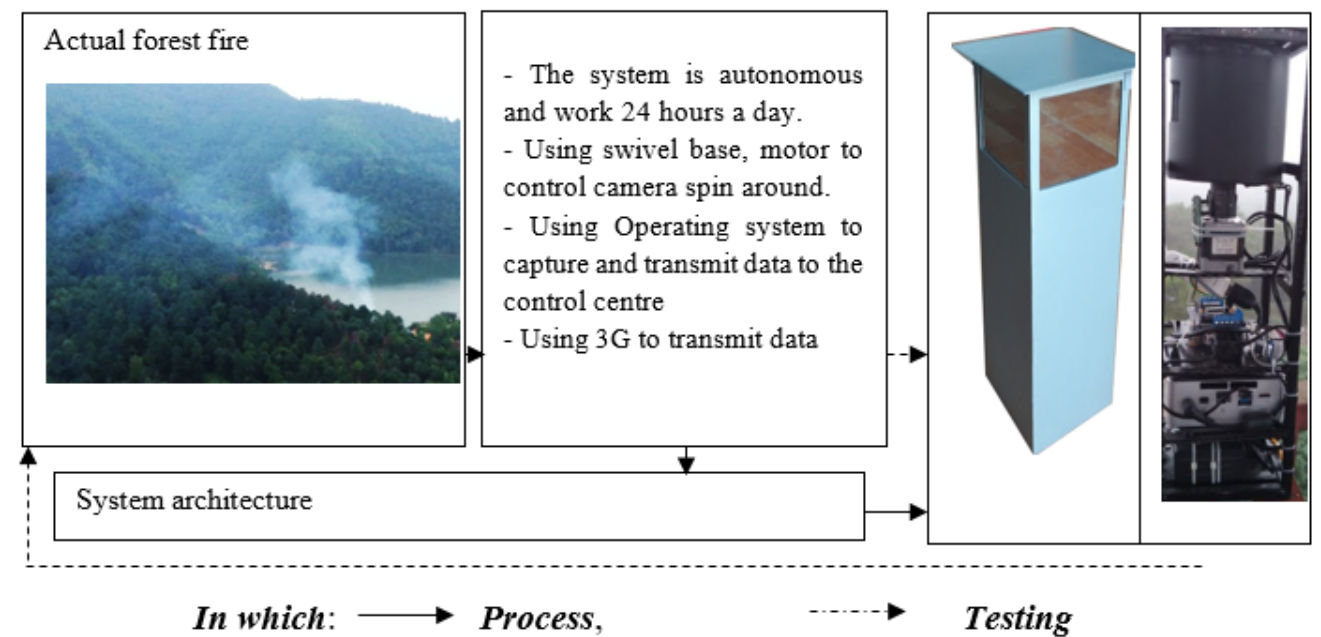

Figure 3 The diagram of the system's function

\subsection{The image-based fire detection software}

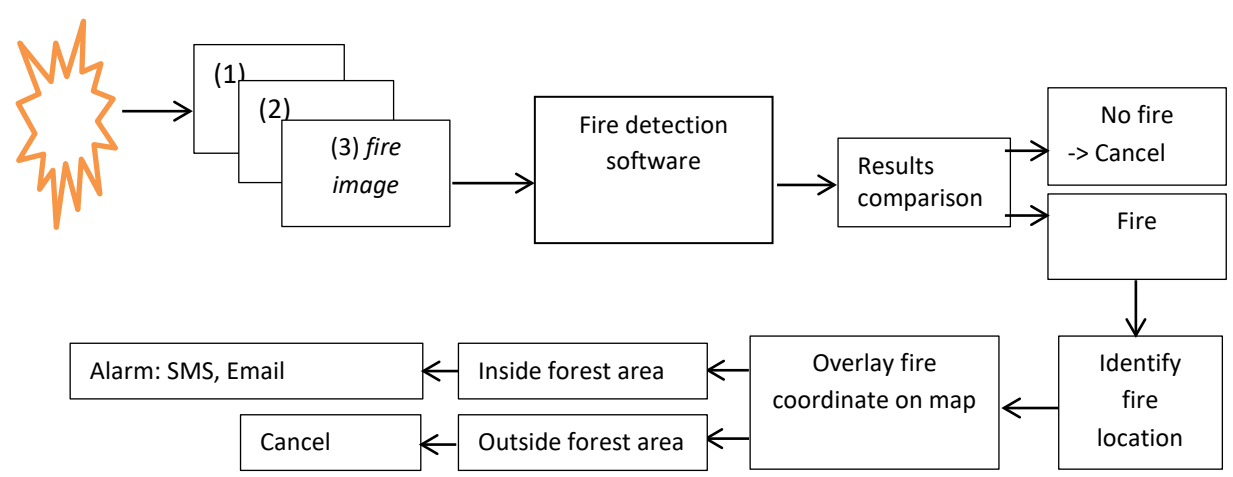

Figure 4. Block diagram of proposed fire detection software

At the same location, camera captured a number of images in different time which are processing and comparing with each other by detection algorithms to give a final result. The coordinate of fires is determined by the two integrated camera. These coordinate are overlaid with forest map, road map and hydrograph map to classify it belong to forest area or not. If the fire occurs in forest area, a firealarming is given immediately through email and SMS. 
Combination of software and hardware in the system is illustrated in figure. 5.

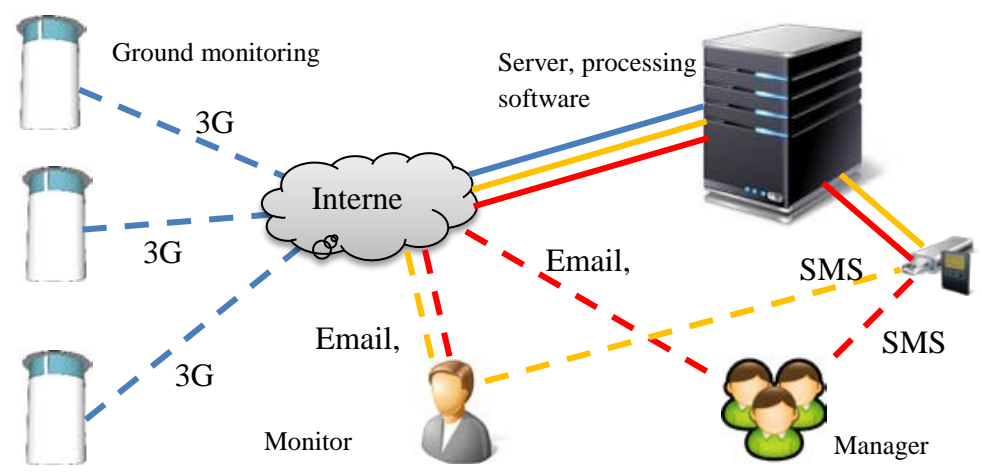

Figure 5. The forest fire detection system architecture

\section{$2.3 \quad$ 2.4. System operation}

Each monitoring system include 01 IP camera connected to the Internet to transmit to the server by 3G signal. These data are captured images which after a predetermined period of time are retrieved automatically by software from serve for analyzing process. In the case of many stations connected to sever, this software will set up the time for assess to station in a fixed cycle that all images could be collected. There more stations connected to server the more operation in the system and the cycle is more complicated.

Each captured images will be analyzed to detect smoke and flame by some smoke or fire detecting algorithms (fig vi). When a fire is detected, system will will identify the exact it's location and give an alarm. The coordinate of a fire can be determined by coordinated of 2 monitoring stations and 2 observation angles from camera in these stations. By overlaying fire's coordinates on forest map, software will define exactly where fire occur in forest or non forest. When detected an abnormality (flame, smoke) the system will give a message to phone, email or website to warning users automatically. The manager can access and chose appropriate solutions.

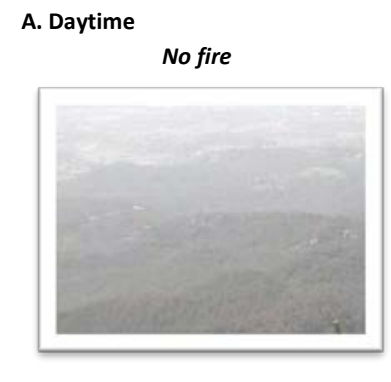

B. Nighttime

No fire

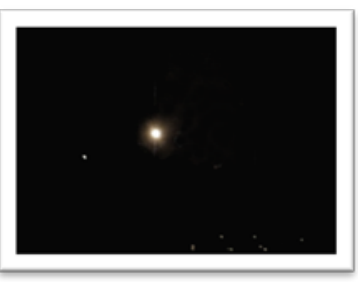

Compared image in software

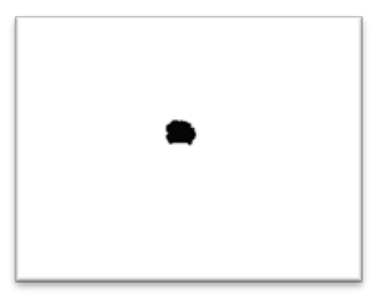

Compared image in software
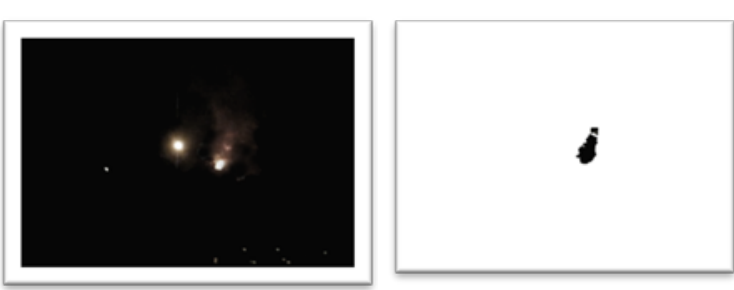

Figure 6. Captured images from camera to determined fire 
Time for analyzing an image is about 0.3 second. Time for loading an image from station depends on the connection speed of $3 \mathrm{G}$ network. However, the image resolution is low ( $280 \times 720$ Pixel- up space of about $140 \mathrm{~Kb}$ ), loading image is not taking too long time. Hence, a camera will load about 1440 images approximate $197 \mathrm{Mb}$ in a day. These images are stored in hardware and will be delete after period of time depend on the space of hardware.

\section{$2.4 \quad$ 2.5. Materials}

The performance of smoke, flame detection and the accuracy of proposed algorithm is evaluated by using different sample video sequences and conducted some "control burnings".

Table 1. The description some of video sequences used in evaluation of the proposed algorithm

\begin{tabular}{|c|l|l|}
\hline TT & \multicolumn{1}{|c|}{ Content } & \multicolumn{1}{|c|}{ Quality } \\
\hline 1 & $\begin{array}{l}\text { The vertical background is crowded with vehicles, } \\
\text { near camera has a large branch of tree that } \\
\text { fffected by strong winds. }\end{array}$ & $\begin{array}{l}\text { - Video size: } 320 \times 240 \text { pixel } \\
\text { - Frame speed: } 9 \mathrm{fps} \\
\text { - Time: 00:11:02 }\end{array}$ \\
\hline 2 & $\begin{array}{l}\text { View from the hill to around in sunny condition, } \\
\text { faint image, effected by insect, weak wind. }\end{array}$ & $\begin{array}{l}\text { - Video size: } 352 \times 288 \text { pixel } \\
\text { - Frame speed: } 25 \mathrm{fps} \\
\text { - Time: 00:04:01 }\end{array}$ \\
\hline 3 & $\begin{array}{l}\text { The frame of a fire in sunny conditions, the location } \\
\text { of fire coincides with horizon, slow fire speed, weak } \\
\text { wind. }\end{array}$ & $\begin{array}{l}\text { - Video size: } 720 \times 576 \text { pixel } \\
\text { - Frame speed: 7fps } \\
\text { - Time: 00:02:01 }\end{array}$ \\
\hline 4 & $\begin{array}{l}\text { The frame of smoke is produced by a factory in } \\
\text { residential area which is crowded with vehicles and } \\
\text { people, weak wind. }\end{array}$ & $\begin{array}{l}\text { - Video size: } 720 \times 576 \text { pixel } \\
\text { - Frame speed: } 10 \mathrm{fps} \\
\text { - Time: 00:01:00 }\end{array}$ \\
\hline 5 & $\begin{array}{l}\text { A frame contains a "control burning" in closely } \\
\text { distance, people walked around the fire. }\end{array}$ & $\begin{array}{l}\text { - Video size: } 320 \times 240 \text { pixel } \\
\text { - Frame speed: } 15 \mathrm{fps} \\
\text { - Time: 00:00:47 }\end{array}$ \\
\hline 6 & $\begin{array}{l}\text { A frame contains a forest fire in closely distance, } \\
\text { the image of the fire covered the frame. }\end{array}$ & $\begin{array}{l}\text { - Video size: } 400 \times 256 \text { pixel } \\
\text { - Frame speed: } 15 \mathrm{fps} \\
\text { - Time: 00:00:13 }\end{array}$ \\
\hline
\end{tabular}

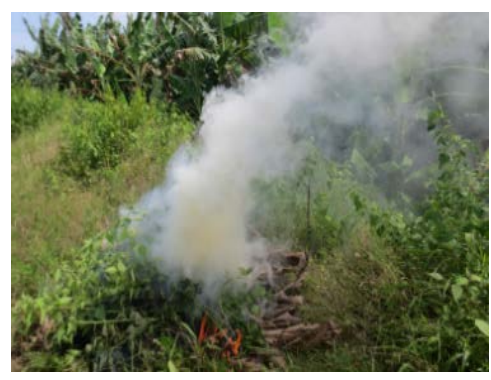

a. U Minh Thuong National Park

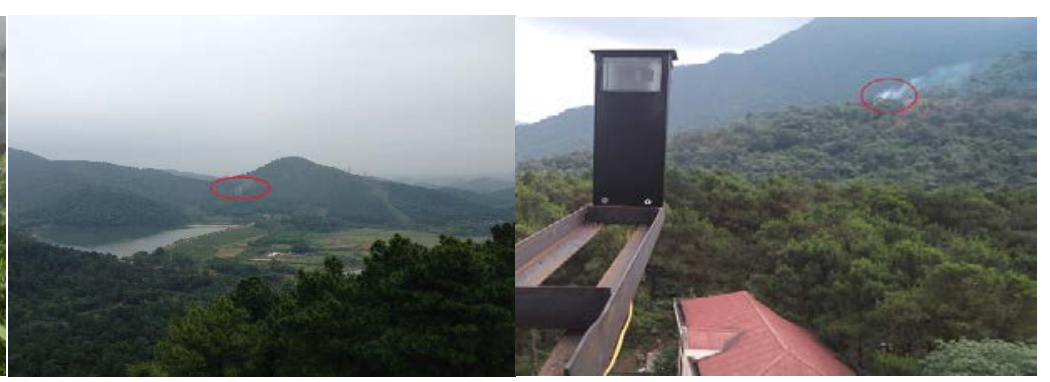

b. Soc Son - Ha Noi c. Ba Vi National Park

Figure 7: The test image of control burning to evaluate the smoke and fire detection capabilities of proposed algorithm

\section{Results and discussions}

\subsection{Testing proposed algorithm with video}

The results of testing some video sequences indicates the performance of the proposed algorithm in detecting smoke and fire. 
Tran Quang Bao, Nguyen Thi Hoa; Approach to Detecting Forest Fire by Image Processing Captured from IP Cameras. Advances in I mage and Video Processing, Volume 5 No 5, October (2017); pp: 1-10

Table 2 The results of evaluating the accuracy of proposed algorithm in video sequences

\begin{tabular}{|c|c|c|c|c|c|c|c|c|c|c|c|c|}
\hline & \multicolumn{2}{|c|}{ Video } & \multicolumn{2}{|c|}{ Video 2 } & \multicolumn{2}{c|}{ Video } & \multicolumn{2}{c|}{ Video 4 } & \multicolumn{2}{c|}{ Video 5 } & \multicolumn{2}{c|}{ Video 6 } \\
\cline { 2 - 14 } No & Detected & $\begin{array}{c}\text { Notec } \\
\text { ted }\end{array}$ & $\begin{array}{c}\text { Detec } \\
\text { ted }\end{array}$ & $\begin{array}{c}\text { No } \\
\text { detec } \\
\text { ted }\end{array}$ & $\begin{array}{c}\text { Detec } \\
\text { ted }\end{array}$ & $\begin{array}{c}\text { No } \\
\text { detec } \\
\text { ted }\end{array}$ & $\begin{array}{c}\text { Detec } \\
\text { ted }\end{array}$ & $\begin{array}{c}\text { No } \\
\text { detec } \\
\text { ted }\end{array}$ & $\begin{array}{c}\text { Detec } \\
\text { ted }\end{array}$ & $\begin{array}{c}\text { Notec } \\
\text { ted }\end{array}$ & $\begin{array}{c}\text { Detec } \\
\text { ted } \\
\text { detec } \\
\text { ted }\end{array}$ \\
\hline 1 & 532 & 3 & 180 & 0 & 49 & 0 & 24 & 0 & 130 & 0 & 154 & 0 \\
\hline 2 & 337 & 6 & 210 & 1 & 65 & 1 & 38 & 0 & 112 & 0 & 128 & 0 \\
\hline 3 & 465 & 22 & 191 & 0 & 51 & 0 & 39 & 0 & 168 & 0 & 153 & 0 \\
\hline 4 & 531 & 7 & 208 & 1 & 39 & 1 & 35 & 1 & 132 & 0 & 154 & 0 \\
\hline 5 & 531 & 6 & 177 & 0 & 35 & 0 & 32 & 1 & 149 & 0 & 130 & 0 \\
\hline 6 & 531 & 7 & 147 & 0 & 32 & 1 & 21 & 0 & 151 & 0 & 151 & 0 \\
\hline 7 & 401 & 25 & 206 & 0 & 21 & 0 & 21 & 0 & 162 & 1 & 154 & 0 \\
\hline 8 & 332 & 36 & 226 & 1 & 21 & 0 & 21 & 0 & 145 & 0 & 154 & 0 \\
\hline 9 & 216 & 6 & 238 & 1 & 23 & 0 & 23 & 0 & 122 & 0 & 154 & 0 \\
\hline 10 & 314 & 8 & 220 & 0 & 29 & 0 & 29 & 1 & 156 & 1 & 154 & 0 \\
\hline Ave. & 419 & 12.6 & 200.3 & 0.2 & 36.5 & 0.82 & 28.3 & 1.06 & 142.7 & 0.2 & 148.6 & 0 \\
\hline
\end{tabular}

Table 2 shows that there is only video 01 have got a false alarm (3\%), and the number of video remaining perform the rate of detecting that approach to $100 \%$ in term of accuracy. There are some reasons that affect on false alarm in video 01. Firstly, in the video many vehicles are running in the road. The second reason is near the camera has a large branch of tree that affected by winds. In contrast, the remaining video, the objects are in static state and clearly background. This indicates that apply proposed algorithm in detecting smoke and fire is effectiveness.

\subsection{Testing proposed algorithm with reality fire}

The yield of proposed algorithm is calculated based on "control burning" in some areas. Fig 07 and 08 show the video sequences in evaluate performance of detecting smoke and fire algorithm, Fig (a1, a2, $a 3, a 4$ and b1, b2, b3, b4) are the frame of fire and Fig $(c 1, c 2, c 3, c 4)$ are images after processed.

- Smoke detection results

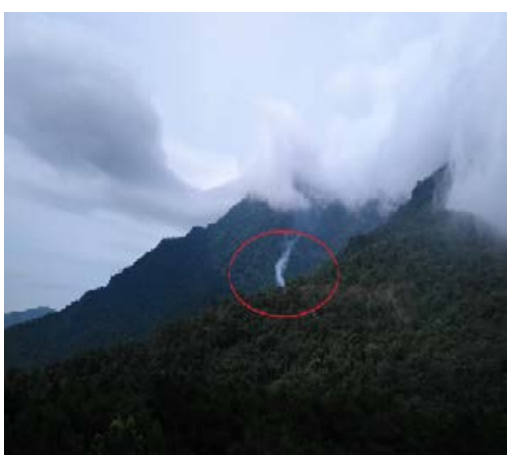

a1

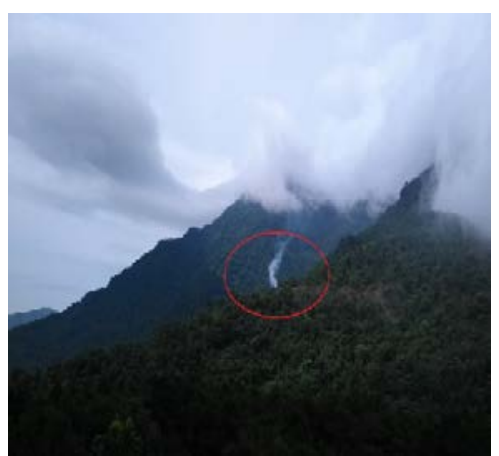

b1

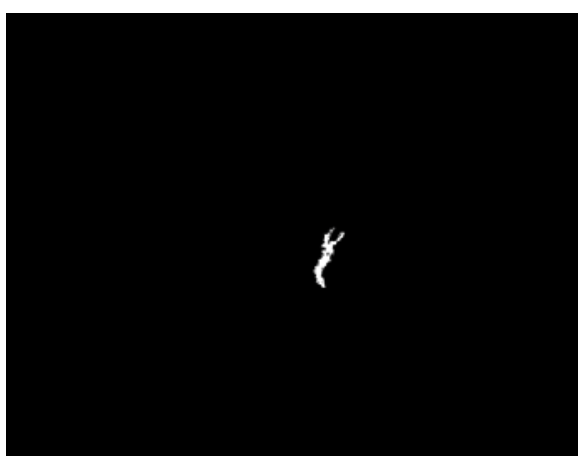

c1

Control burning in Ba Vi National park 


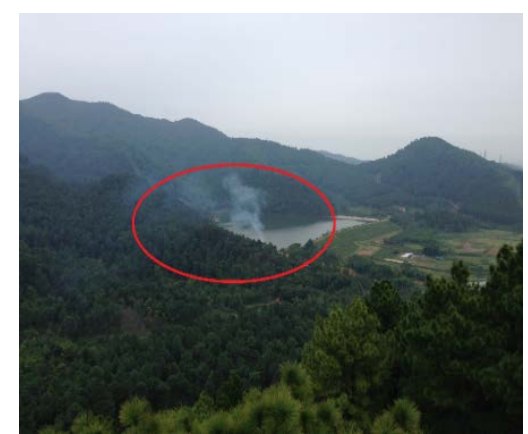

a2

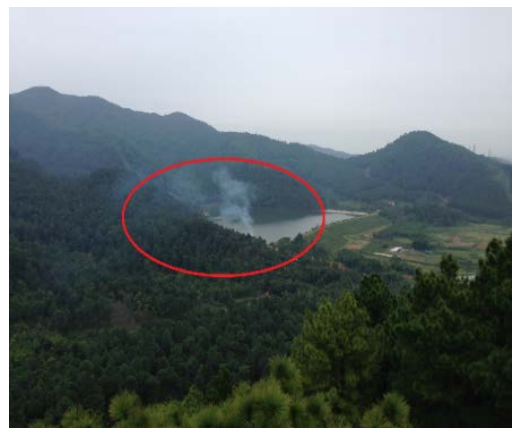

b2

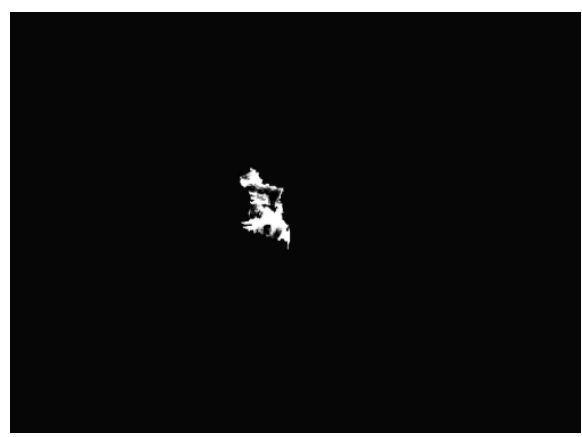

c2

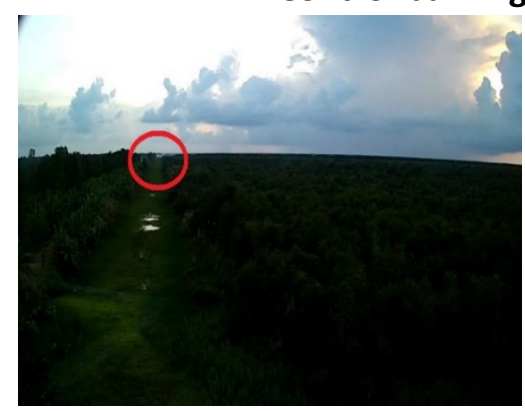

a3

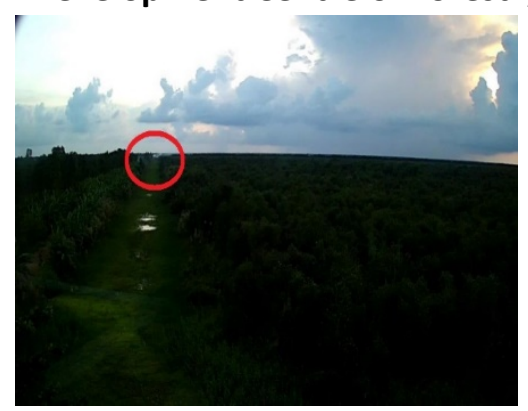

b3

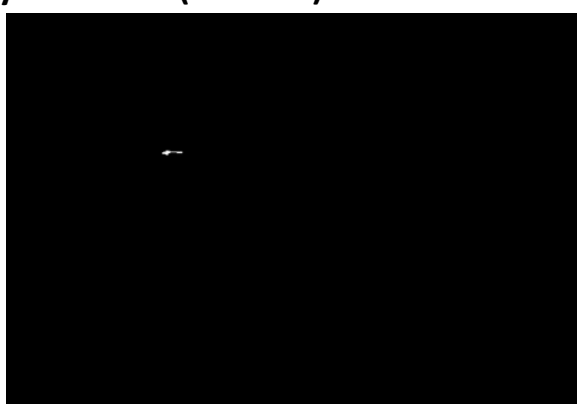

c3

Control burning in U Minh Thuong National Park

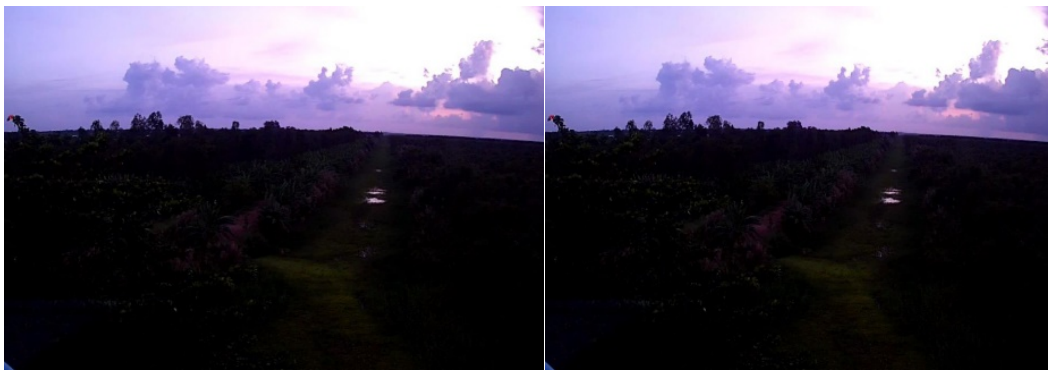

a4

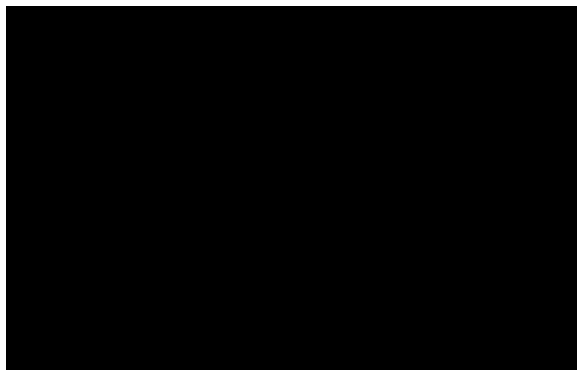

c4

Control burning in U Minh Thuong National Park

Figure 8. Smoke detection performance; $\left(a_{i}, b_{i}\right)$ Frame sequences; $\left(c_{i}\right)$ image after processing

- Fire detection results (night time)

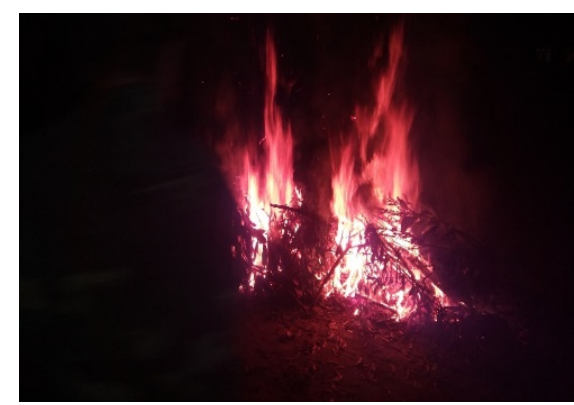

a

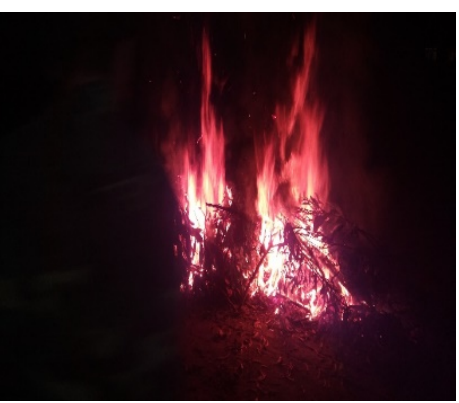

b

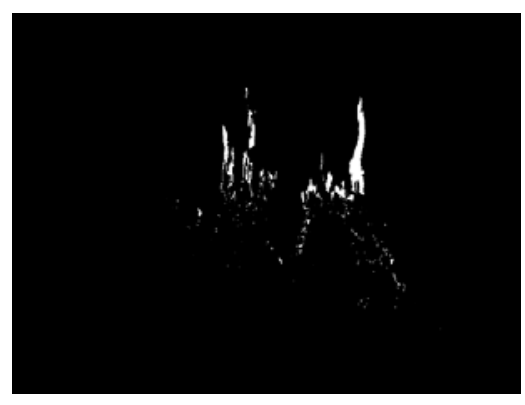

C

Figure 9. Fire detection performance; $(a, b)$ video sequences; $(b)$ image after processing.

Performance results on video sequences show that there are to cases including detected or nondetected. In the second case, smoke or fire cannot be detected because of many different reasons such as the color of background and smoke/fire is the same, and the observation distance from camera effect on the accuracy (fig viii - a4, b4, c4). 


\section{Conclusions}

The Discrete Cosine Transform (DCT) algorithm of each $8 \times 8$ block is the input for smoke processing and fire detection.

The characteristics of smoke, fire, motion, color and expansion are analyzed directly in the DCT domain to minimize the time and increase the accuracy of the results.

The DCT algorithm used video sequences and control burning, the results show the accuracy of detection up to $97 \%$. The main cause of false detection is the similarity of color between background and smoke, the distance of the camera to the fire location.

The JPEG format processing algorithm from IP Camera can be applied efficiently in early detection of forest fires in Vietnam.

Application software for early detection of forest fires from the ground observation station is designed and developed based on the requirements of forest resource management and minimization of forest fire damage in areas where fire occurs frequently in Vietnam.

The software is integrated with ground observation stations, regular monitoring stations and fire monitoring by IP cameras. The monitoring stations are compact and stable designed with low cost and efficient for forest fire monitoring tasks in Vietnam.

\section{REFERENCES}

[1] Chen, T.; Yin, S; Huang, Y.; Ye, Y. The Smoke Detection for Early Fire-Alarming System Based on Video Processing. In Proceedings of International Conference on Intelligent Information Hiding and Multimedia Signal Processing, Pasadena, CA, USA, 18-20 December 2006.

[2] Leonardo Millan-Garcia, Gabriel Sanchez-Perez, Mariko Nakano, Karina Toscano-Medina, Hector Perez-Meana and Luis Rojas-Cardenas, "An Early Fire Detection Algorithm Using IP Cameras" in Sensors 2012, 12, 5670-5686, pp. 5670-5686.

[3] Schneiderman, R. Trends in Video Surveillance Given DSP an Apps Boost. IEEE Sign. Process. Mag. 2010, 6, 6-12.

[4] Turgay Çelik, Hüseyin Özkaramanlı, and Hasan Demirel, "Fire and smoke detection without sensors: Image processing based approach" in 15th European Signal Processing Conference (EUSIPCO 2007), Poznan, Poland, September 3-7, 2007, pp. 1794-1798.

[5] Yuan F. Video-Based Smoke Detection with Histogram Sequence of LBP and LBPV Pyramids. Fire Safety J. 2011, 46, 132-139.

[6] Yu C.; Faon J.; Wang J.; Zhang Y.; State K. Video Fire Smoke Detection Using Motion and Color Features. Fire Technol. 2010, 46, 651-663. 